\title{
Discovering the Use of a Home Smart Telephone: A Persona Approach
}

\author{
Weijane Lin, Chih-Lo Chen, Chien-Ting Yang, and Hsiu-Ping Yueh \\ Department of Library and Information Science, National Taiwan University \\ Department of Bio-Industry Communication and Development, National Taiwan University \\ No. 1., Sec. 4., Roosevelt Rd., Daan Dist., Taipei 10617, Taiwan, R.O.C. \\ vjlinentu.edu.tw
}

\begin{abstract}
This ongoing study examined the users' needs, perceptions, performance and expectations toward a multifunctional home-use smart telephone. Through systematically discovering users' behaviors, this study investigated 317 participants' use of the target product, and profiled the target users of personas within different scenarios. Three major patterns of use were found in this study, and the suggestions were made to improve the interface and marketing strategies with references to the personas concluded in this study.
\end{abstract}

Keywords: Home phone, Smartphone, Persona.

\section{Introduction}

With the common ownership of home-use telephone in households in many developed countries, the use of a domestic telephone involved a variety of everyday living activities that were communicational, informational, recreational and even safetyrelated. According to a governmental statistics (2012) in Taiwan, although the owning rate was declining accompanied with the growing ownership of mobile phones and broadband networks, averagely every household owned 1.2 home-use telephone in the past decade. The availability of home-use telephone has made it a significant device in national information and communication infrastructure. The functions and the services available by home-use telephones have been rapidly and extensively developed with making alliances with telecommunication, broadcasting, medical and social networks by the assemblages of ICT technologies. However, while more functions were integrated into an originally single-purposed appliance, the users might be overwhelmed by the unfamiliar conceptual models thus hampered their acceptance and performance of using it. Consequently, to understand users' requirements and behaviors in advance would be necessary for such a product to be developed and implemented. In this vein, this study examined the users' needs, perceptions, performance and expectations toward a multifunctional home-use smart telephone to discover their use by the systematically presentation of personas.

While the user-centered design approach gained more and more attention in product development, the difficulties remained in practices when the design teams intended to include the actual users. The needs and goals of users were not always 
communicated effectively between the designers and the users. Misunderstanding occurred in communications due to various levels of expertise and experiences, and it was not easy to leverage the features of the product between the satisfactory experiences of all users. The idea of persona that coined by Cooper (1999), served as a design target, a communication tool and an instrumental method that widely acknowledged by the industry.

Following the user-centered design principles, personas facilitated designers to construct the detail and image of target users by drawing characters in order to design a product that satisfied the user needs (Pruitt \& Adlin, 2006). It referred to the archetypal character that represented a group of users who share common goals, attitudes and behaviors when interacting with a product or service. A persona served as a fictitious user for designers, and facilitated them make difficult decisions from the users' perspectives during the product design and development (Chang, Lim, \& Stolterman, 2008). In addition to a general and broad image of the user population, to construct a persona involved qualitative and quantitative collection of data to profile real people in order to discover their own needs, motivations and goals in details (Idoughi, Seffah, \& Kolski, 2012). The data was analyzed and integrated by a contextual presentation that incorporated the scenarios, backgrounds, behaviors and emotions of users when interacting with the product (Lester, Converse, Kahler, Barlow, Stone, \& Bhogal, 1997).

By adopting the persona-based approach, a practical goal of this study is providing the designers with the genuine life experiences of users to develop products and services that meet their needs. Comparing to the previous design practices and studies, in which designers misunderstood the users' thoughts to be similar to theirs, and the failing pursuit to design a perfect product that satisfied every user, personas authentically reflected different users, whether novice or expert, and revealed the obstacles and problems they had encountered (Lindgren, Chen, Amdahl, \& Chaikiat, 2007). Personas suggested not an average model of users, but a context-rich user scenario. Additionally, personas that contained rich information of target users also served experiential reference for designers to evaluate the usability of the products, and provided insightful information to form marketing strategies (Cooper, Reimann, \& Cronin, 2012).

This study aimed to discover the use of a home-use smartphone by the personabased approach. The overall goals of this study are:

1. Investigate the users' perceptions and performances with using the target product of a home phone.

2. Incorporate persona to profile and present the target users of a multifunctional home phone.

3. Explore the implications of the interface and functionality design facing the developing of a multifunctional home phone.

\section{Methodology}

In this study a commercial product of a smart home-use telephone provided by a telecommunications company in Taiwan was subjected as the target product. And the 
personas that use this target product were developed. For a real project and the commercial product, applying personas that consisted of widely collected user data would help the designers better understand the users' requirements. Additionally, through the thorough investigation of the target users, potential markets and users groups could be found.

Followed the principles of constructing personas, to understand the user experience of the home-use smart telephones and the characteristics of different user groups, both quantitative and qualitative data from multiple sources was collected. These sources included the focus group interview of heavy users, the articles post on the online user community where experiences of using home-use smart telephone were shared. Additionally, literatures and reports regarding specific user groups' life and consumer behaviors were also referred.

This study adopted the purposeful sampling strategy to recruit 317 experienced users in total to participate in this study. According to their frequency and familiarity with the target product, 7 participants were selected for a focus group interview, while 310 of them were interviewed by phone with a structural survey about their use. Based on the analysis of these participants' use behaviors, personas for the target product were developed. These personas detailed different purposes and scenarios of use, and specifically this study took four major steps until the personas were developed.

1. Documents analysis: Qualitative investigation including the collection of opinions regarding the target product in several online user communities and the analysis of the official documents was conducted.

2. Focus group interview with the heavy users of the target product: 7 users were selected as the subjects in this stage by their product use logs. Sample questions of the interview included the reason of purchasing the target product, the benefits and challenges of using the target product, the functions and services of frequent and rare use.

3. User Survey: 310 interviewees were sampled from the existing users of the target product, and interviewed by phone with a structural questionnaire that developed in house by the researcher. The instrument consisted of four major aspects that investigated the users' purposes, frequencies, perceptions and expectations of each function and service provided by the target product.

4. Development of personas: With reference to the findings of the previous steps, personas that described the target users were developed accordingly.

\section{Preliminary Results}

Among the 7 users participated in the focus group interview, 4 were males and 3 were females. Their age ranged from 31 to 70 , all have the target product installed at home for more than 1.5 years. The major reason of purchased is for the convenience, novelty and the trust in the branding of the telecommunications company. Most of the users compared the target product with the computers and regarded it as more intuitive to use without complex prerequisites. 
The functions that frequently used by the users were largely accessing daily life information including the weather reports, lottery, local maps and hospitals. While the other major functionalities that emphasized by the stakeholders, the surveillance and videoconferencing services, were rarely used by the interviewees. This found gap also revealed that the target consumers of the product were not the major users of the product.

The findings of the survey that targeted the 310 current users suggested that the major user population were the elderly couples, which echoed the results of the focus group interview. Users in nuclear family style, who were young couples with one or two children were the secondly frequent users of the target product. Based on the users' purposes that derived from the preliminary findings, 3 personas were developed to accumulate the large amount of real user data and represent the target users' profiles.

\subsection{Persona A: Use for Daily Life Information}

The first persona represented the user population of housewives whose major purpose of the target product was to access daily life information. Compared to other consumer-electronics, she is more satisfied with the interface of the home-use smart telephone. In order to depict the scenario of requiring daily life information by using the home-use smart telephone, we searched for the empirical studies about the information needs, hobbies, and consumer behaviors of housewives and women who are retired.

The scenario of use suggested that the users could access weather, calendar and security information readily and conveniently at the door when they went out, leisurely check the sales information with the music played on, and made reservations with the transportation services. The information and services the users expected for a homeuse smart telephone were often connected with other home appliances and activities such as television, intercoms, newspapers and magazines.

\subsection{Persona B: Use for Communication}

This persona represented the middle-aged who use the target product for contacting family members for caring and greeting purposes. Theoretical and practical references about the family communication and the facilities of elder caring and healthcare were referred to design the scenario for this persona. The users could make video phone calls easily in more casual settings, and with integrative use of the surveillance functions, the users valued the home-use smart telephone more than mobile phones.

The scenario of use also suggested that the interface of the home-use telephone served the functionality of videoconferencing better than television and computer. Telephone was regarded usable for various users including the elders and the children, and therefore served better for communication purposes.

\subsection{Persona C: Use for Surveillance and Security System}

According to the findings of the interviews, the target product was then compared to other products to discover the advantages and weakness of it. And by referring to the 
consumer studies of security products, the persona $\mathrm{C}$ was developed to represent users who seek for affordable solutions of home security. The simplified functionality of surveillance satisfied the users by offering alarming and recording services.

\section{Discussion and Further Investigation}

The current study examined the users' needs, perceptions, performance and expectations toward a home-use smart telephone. Through the preliminary investigations, three personas were developed with references to qualitative and quantitative materials that profiled the users. By adopting the persona approach, it was possible to provide a vivid and accurate representation of the target users, and communicate the users' needs and goals more effectively with the stakeholders.

The preliminary findings of the study supported that the residential home phone represented differently in its nature from smartphones, and suggested different user expectations and experiences. For endeavors that intended to extend the functionality of the appliance, or to enable residential home phone of more integrative services with other home appliances, it was noteworthy to consider the conceptual models discovered in this study. With reference to the personas developed by this current study, effective and efficient user experiments could be conducted continuously under the designated scenario to drive and validate the stakeholders' and users' concerns about the product design.

\section{References}

1. Chang, Y.N., Lim, Y.K., Stolterman, E.: Personas: from theory to practices. In: Proceedings of the 5th Nordic Conference on Human-computer Interaction: Building Bridges, pp. 439$442(2008)$

2. Cooper, A.: The Inmates are Running the Asylum. Macmillan Publishing Company Inc., New York (1999)

3. Cooper, A., Reimann, R., Cronin, D.: About Face 3: The Essentials of Interaction Design. Wiley Publishing, Indianapolis (2012)

4. Lindgren, A., Chen, F., Amdahl, P., Chaikiat, P.: Using personas and scenarios as an interface design tool for advanced driver assistance systems. In: Stephanidis, C. (ed.) UAHCI 2007 (Part II). LNCS, vol. 4555, pp. 460-469. Springer, Heidelberg (2007)

5. Pruitt, J., Adlin, T.: The Persona Lifecycle: Keeping People in Mind throughout Product Design. Morgan Kaufmann Publishers (2006)

6. Lester, J.C., Converse, S.A., Kahler, S.E., Barlow, S.T., Stone, B.A., Bhogal, R.S.: The persona effect: affective impact of animated pedagogical agents. In: Proceedings of the SIGCHI Conference on Human Factors in Computing Systems, pp. 359-366 (1997)

7. Idoughi, D., Seffah, A., Kolski, C.: Adding user experience into the interactive service design loop: a persona-based approach. Behaviour \& Information Technology 31(3), 287-303 (2012) 